\title{
SPHEREx: an all-sky NIR spectral survey
}

\section{Phillip M. Korngut, James J. Bock, Rachel Akeson, Matthew Ashby, Lindsey Bleem, et al.}

Phillip M. Korngut, James J. Bock, Rachel Akeson, Matthew Ashby, Lindsey Bleem, Justin Boland, Douglas Bolton, Samuel Bradford, David Braun, Sean Bryan, Peter Capak, Tzu-Ching Chang, Andrew Coffey, Asantha Cooray, Brendan Crill, Olivier Doré, Tim Eifler, Chang Feng, Salman Habib, Katrin Heitmann, Shoubaneh Hemmati, Christopher Hirata, Woong-Seob Jeong, Minjin Kim, Davy Kirkpatrick, Theresa Kowalkowski, Elisabeth Krause, Carey Lisse, Philip Mauskopf, Daniel Masters, James McGuire, Gary Melnick, Hein Nguyen, Hooshang Nayyeri, Karin Oberg, Roland dePutter, William Purcell, Jennifer Rocca, Marcus Runyan, Karin Sandstrom, Roger Smith, Yong-Seon Song, Nathaniel Stickley, Jeremy Stober, Sara Susca, Harry Teplitz, Volker Tolls, Stephen Unwin, Michael Werner, Rogier Windhorst, Michael Zemcov, "SPHEREx: an all-sky NIR spectral survey," Proc. SPIE 10698, Space Telescopes and Instrumentation 2018: Optical, Infrared, and Millimeter Wave, 106981U (6 July 2018); doi: 10.1117/12.2312860 


\title{
SPHEREx: an all-sky NIR spectral survey
}

Phillip M. Korngut ${ }^{\mathrm{a}}$, James J. Bock ${ }^{\mathrm{a}, \mathrm{e}}$, Rachel Akeson ${ }^{\mathrm{b}}$, Matthew Ashby ${ }^{\mathrm{c}}$, Lindsey Bleem ${ }^{\mathrm{d}}$, Justin Boland ${ }^{\mathrm{e}}$, Douglas Bolton ${ }^{\mathrm{e}}$, Samuel Bradford ${ }^{\mathrm{e}}$, David Braun ${ }^{\mathrm{e}}$, Sean Bryan', Peter Capak $^{\mathrm{a}}$, Tzu-Ching Chang ${ }^{\mathrm{e}}$, Andrew Coffey ${ }^{\mathrm{e}}$, Asantha Cooray ${ }^{\mathrm{f}}$, Brendan Crille, Olivier Dorée, ${ }^{\mathrm{e}}$,

Tim Eiflere, Chang Feng ${ }^{f}$, Salman Habib ${ }^{\mathrm{d}}$, Katrin Heitmann ${ }^{\mathrm{d}}$, Shoubaneh Hemmati ${ }^{\mathrm{b}}$,

Christopher Hirata $^{\mathrm{d}}$, Woong-Seob Jeong ${ }^{\mathrm{h}}$, Minjin Kim ${ }^{\mathrm{h}}$, J. Davy Kirkpatrick ${ }^{\mathrm{b}}$, Theresa

Kowalkowskie ${ }^{\mathrm{e}}$, Elisabeth Krause ${ }^{\mathrm{e}}$, Carey Lisse ${ }^{\mathrm{i}}$, Philip Mauskopf ${ }^{\mathrm{l}}$, Daniel Masters ${ }^{\mathrm{e}}$, James

McGuire $^{\mathrm{e}}$, Gary Melnick ${ }^{\mathrm{c}}$, Hien Nguyen ${ }^{\mathrm{e}}$, Hooshang Nayyeri ${ }^{\mathrm{f}}$, Karin Oberg ${ }^{\mathrm{c}}$, Roland dePutter ${ }^{\mathrm{a}}$, William Purcell ${ }^{\mathrm{j}}$, Jennifer Rocca ${ }^{\mathrm{e}}$, Marcus Runyan ${ }^{\mathrm{e}}$, Karin Sandstrom ${ }^{\mathrm{k}}$, Roger Smith $^{\mathrm{a}}$, Yong-Seon Song ${ }^{\mathrm{h}}$, Nathaniel Stickley ${ }^{\mathrm{b}}$, Jeremy Stober ${ }^{\mathrm{j}}$, Sara Susca ${ }^{\mathrm{e}}$, Harry Teplitz ${ }^{\mathrm{b}}$, Volker Tolls ${ }^{\mathrm{c}}$, Stephen Unwin ${ }^{\mathrm{e}}$, Michael Werner ${ }^{\mathrm{e}}$, Rogier Windhorst ${ }^{\mathrm{l}}$, and Michael Zemcov ${ }^{\mathrm{m}, \mathrm{e}}$

${ }^{a}$ California Institute of Technology, 1200 E. California Blvd, Pasadena, CA 91125, USA

${ }^{b}$ IPAC, Caltech, 770 S. Wilson Ave, Pasadena, CA 91125, USA

c Harvard Smithsonian CfA, 60 Garden St., Cambridge, MA 02138, USA

${ }^{\mathrm{d} A r g o n n e}$ National Laboratory, High-Energy Physics Division, 9700 S. Cass Avenue, Argonne, IL 60439,USA

e Jet Propulsion Laboratory, 4800 Oak Grove Drive, Pasadena, CA 91109, USA

${ }^{f}$ University of California Irvine, 4186 Frederick Reines Hall, Irvine, CA 92697, USA

'Department of Astronomy, The Ohio State University, 140 W. 18th Avenue, Columbus, OH 43210 USA

${ }^{\mathrm{h}}$ Korea Astronomy and Space Science Institute, Daejeon, 34055, Korea

${ }^{i}$ JHU-APL, SES/SRE, Bldg 200/E206, 11100 Johns Hopkins Road, Laurel, MD 20723, USA

${ }^{j}$ Ball Aerospace Corporation, 1600 Commerce Street, Boulder, CO 80301 USA

${ }^{k}$ University of California, 9500 Gilman Drive, San Diego, La Jolla, CA 92093, USA

${ }^{1}$ Arizona State University, Department of Physics, P.O. Box 871504, Tempe, AZ 85287, USA ${ }^{m}$ Rochester Institute of Technology, College of Science, 74 Lomb Memorial Drive, Rochester,

NY 14623,USA

\begin{abstract}
SPHEREx, a mission in NASA's Medium Explorer (MIDEX) program recently selected for Phase-A implementation, is an all-sky survey satellite that will produce a near-infrared spectrum for every 6 arcsecond pixel on the sky. SPHEREx has a simple, high-heritage design with large optical throughput to maximize spectral mapping speed. While the legacy data products will provide a rich archive of spectra for the entire astronomical community to mine, the instrument is optimized for three specific scientific goals: to probe inflation through the imprint primordial non-Gaussianity left on today's large-scale cosmological structure; to survey the Galactic plane for water and other biogenic ices through absorption line studies; and to constrain the history of galaxy formation through power spectra of background fluctuations as measured in deep regions near the ecliptic poles. The aluminum telescope consists of a heavily baffled, wide-field off-axis reflective triplet design. The focal plane is imaged simultaneously by two mosaics of H2RG detector arrays separated by a dichroic beamsplitter. SPHEREx assembles spectra through the use of mass and volume efficient linear variable filters (LVFs) included in the focal plane assemblies, eliminating the need for any dispersive or moving elements. Instead, spectra are constructed through a series of small steps in the spacecraft attitude across the sky, modulating the location of an object
\end{abstract}

Further author information: (Send correspondence to P.M.K.)

P.M.K.: E-mail: pkorngut@caltech.edu

Space Telescopes and Instrumentation 2018: Optical, Infrared, and Millimeter Wave, edited by Makenzie Lystrup, Howard A. MacEwen, Giovanni G. Fazio, Proc. of SPIE Vol. 10698, 106981U

(C) 2018 SPIE $\cdot$ CCC code: $0277-786 X / 18 / \$ 18 \cdot$ doi: $10.1117 / 12.2312860$ 
within the FOV and varying the observation wavelength in each exposure. The spectra will cover the wavelength range between 0.75 and $5.0 \mu \mathrm{m}$ at spectral resolutions ranging between $\mathrm{R}=35$ and $\mathrm{R}=130$. The entire telescope is cooled passively by a series of three $\mathrm{V}$-groove radiators below $80 \mathrm{~K}$. An additional stage of radiative cooling is included to reduce the long wavelength focal plane temperature below $60 \mathrm{~K}$, controlling the dark current. As a whole, SPHEREx requires no new technologies and carries large technical and resource margins on every aspect of the design.

Keywords: All Sky survey, linear variable filters, near infrared, cosmology, biogenic ices, extragalactic background light

\section{INTRODUCTION}

SPHEREx is an all-sky survey mission designed to address all three science goals in NASA's astrophysics division:

1. Probe the origin and destiny of our Universe. SPHEREx constrains the physics of inflation, the superluminal expansion of the Universe that took place $\sim 10^{-32} \mathrm{~s}$ after the Big Bang. SPHEREx reveals the imprints of inflation in the three-dimensional large-scale distribution of matter by measuring galaxy redshifts over a large cosmological volume.

2. Explore whether planets around other stars could harbor life. SPHEREx investigates the origin of water and biogenic molecules in the early phases of planetary system formation - from molecular clouds to young stellar systems with planet-forming disks - by measuring absorption spectra to determine the abundance and composition of ices.

3. Explore the origin and evolution of galaxies. SPHEREx charts the origin and history of galaxy formation through a deep mapping survey. This survey measures galaxy clustering to trace the total light produced by all galaxy populations, complementing studies based on deep galaxy counts, to probe the cosmic history of light production, from the first galaxies that ended the cosmic dark ages to the present day.

To accomplish these goals, SPHEREx conducts the first near-infrared all-sky spectral survey useful for a broad range of astrophysics. It will deliver to the community a dataset of lasting legacy in planetary, Galactic, and extragalactic science. Two workshops involving more than 90 scientists from outside the SPHEREx team have defined numerous scientific questions which could be addressed by SPHEREx legacy dataset and also discuss the synergy between SPHEREx and other astronomical facilities of the 2020's. The results of these workshops are reported in 1,2 .

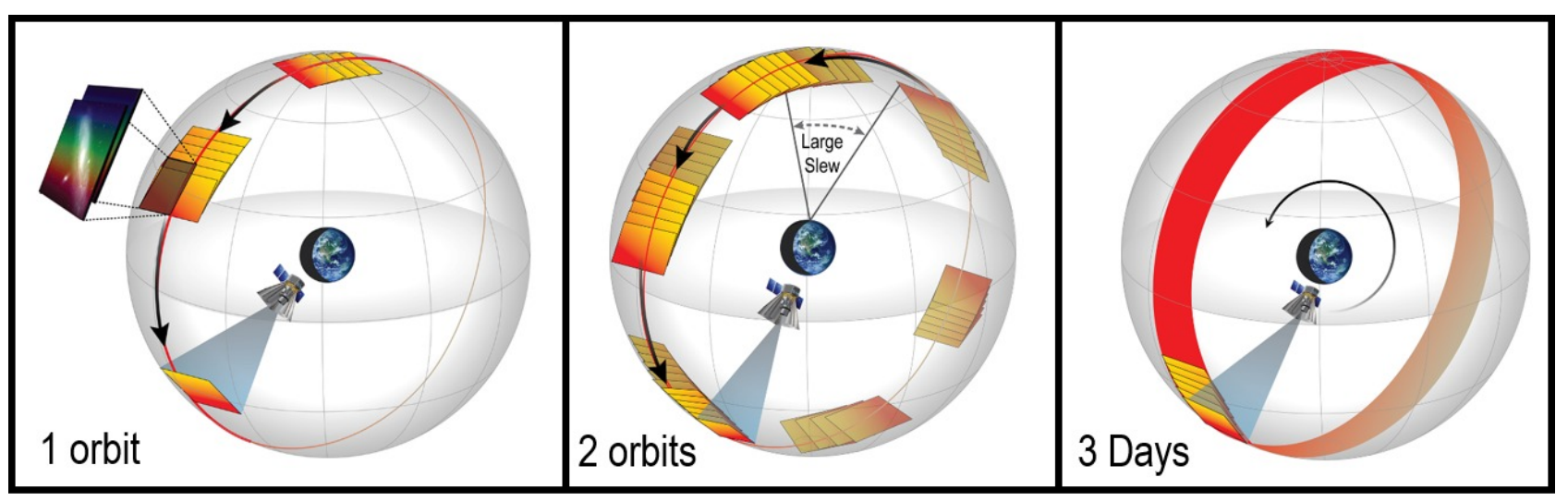

Figure 1. The SPHEREx scan strategy builds up the all sky coverage with a series of large and small slews. These follow a great circle, building a complete spectrum on average every three days. This circle processes as the Earth goes around the Sun, producing a full sky coverage every six months. 
At the core of the survey design are a set of linear variable filters (LVFs) placed $100 \mu \mathrm{m}$ above the telescope's focal planes. These are effectively bandpass filters with central wavelengths that vary as a function of position on the substrate. This focal plane configuration is used in concert with a scanning pattern designed to target each point of the celestial sphere at 96 locations across the field of view. Accomplishing this every six months, SPHEREx produces a contiguous NIR spectrum across the entire sky.

In this paper, we present an overview of SPHEREx's science implementation with a focus on the payload design. Additionally, we report on fabricated prototype performance of key sub-sytems accomplished during the Phase-A effort.

\section{SURVEY OVERVIEW}

SPHEREx adapts the WISE observing strategy, using a $700 \mathrm{~km}$ low-earth sun-synchronous orbit and pointing the spacecraft $\sim 90^{\circ}$ from the Sun, so that observations follow along a great circle that includes the north and south ecliptic poles. Unlike WISE, SPHEREx carries out a sequence of inertial spacecraft pointings producing a series of long $(\sim 150 \mathrm{~s})$ exposures. These enable the background-limited noise performance essential for sensitive spectroscopic observations. To prevent Terrestrial and Solar emission from loading the cold components of the payload, the survey strategy must avoid attitudes $>35^{\circ}$ from local zenith, and $<91^{\circ}$ from the Sun at all times. As the spacecraft orbits the Earth, it executes two types of pointing slews to produce an all-sky survey while maintaining the aforementioned avoidance criteria: large slews up to $70^{\circ}$ and small 12.3 arcminute slews. The large slews allow the spacecraft to "look ahead" in its orbit by up to $35^{\circ}$ so that the telescope can stay inertially pointed for up to 18 minutes without violating Earth and Sun avoidance constraints. After completing a large slew, the spacecraft executes a series of $\sim 150 \mathrm{~s}$ exposures separated by small slews that step along the LVF to fill in the spectral sampling. The 12.3 arcminute small steps correspond to the width of the spectral channels on the LVFs. After 3-5 small slews and exposures, the spacecraft again executes a large slew to maintain Earth/sun avoidance 3. The scan strategy is summarized in Figure (1).

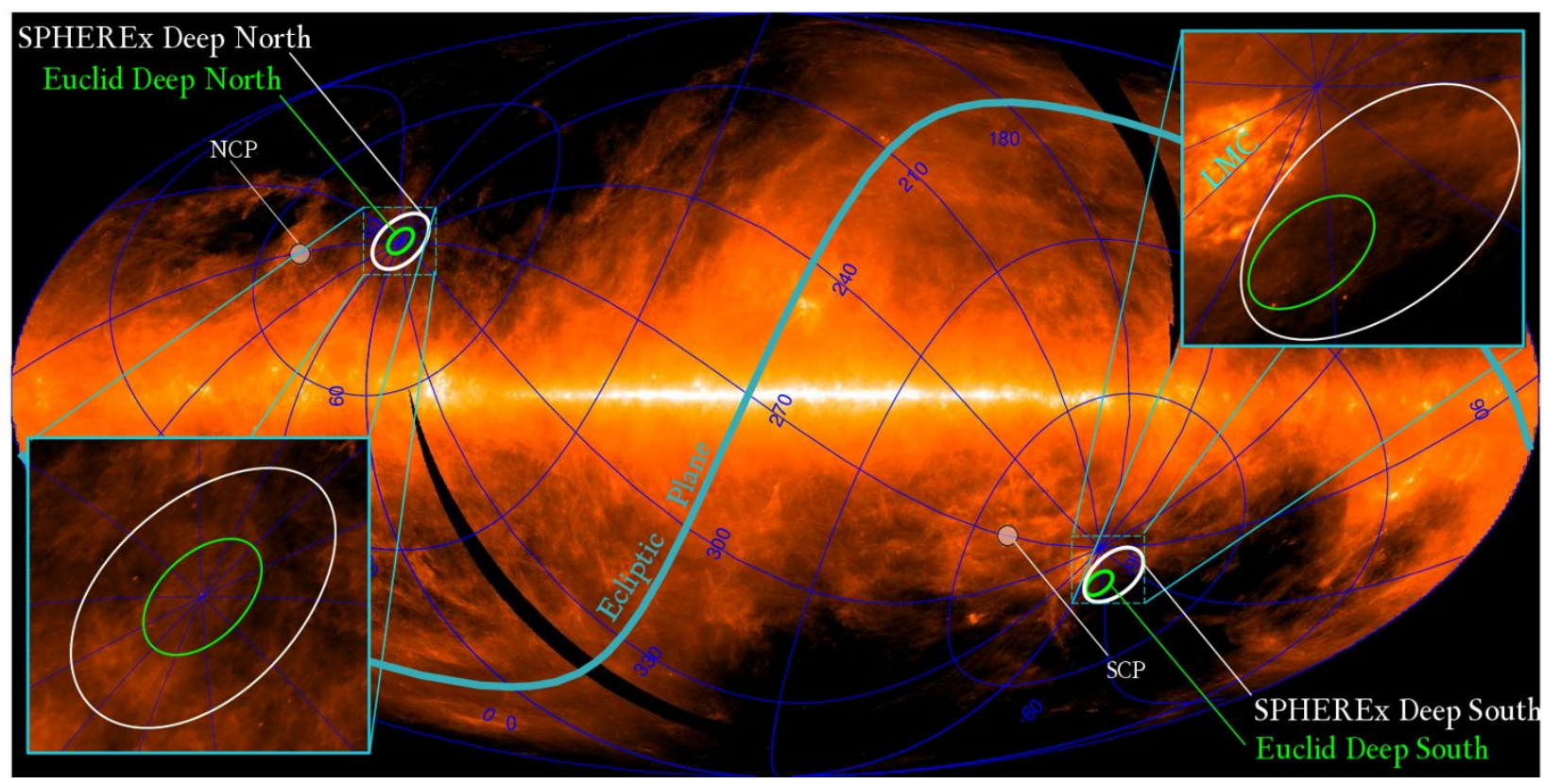

Figure 2. The location of SPHEREx's deep regions at the Ecliptic Poles shown on top of IRAS $100 \mu \mathrm{m}$ dust map. The southern deep field is slightly offset from the ecliptic pole to avoid the Large Magellanic Cloud 4.

The targets generally follow along a great circle, keeping the angle from the spacecraft + z-axis to the sun $>91^{\circ}$. The great circle precesses $\sim 1^{\circ}$ per day. The observing pattern runs by executing a pre-planned sequence of spacecraft slews and pointed exposures that sample every sky position in all spectral bins at least once in each 
survey. The scan naturally crosses near the ecliptic poles each orbit, producing one northern and one southern deep field. The locations of these roughly $100 \mathrm{deg}^{2}$ fields are shown with respect to the Galactic foreground in Figure 2.

Combining mature estimates of other performance drivers (e.g slew times, optical efficiency, photon and read noise, spectral resolution, dark current and pointing stability) with scan strategy parameters; we estimate the projected point source sensitivity given in Figure 3. For reference, this is shown alongside the sensitivities of selected other relevant surveys.

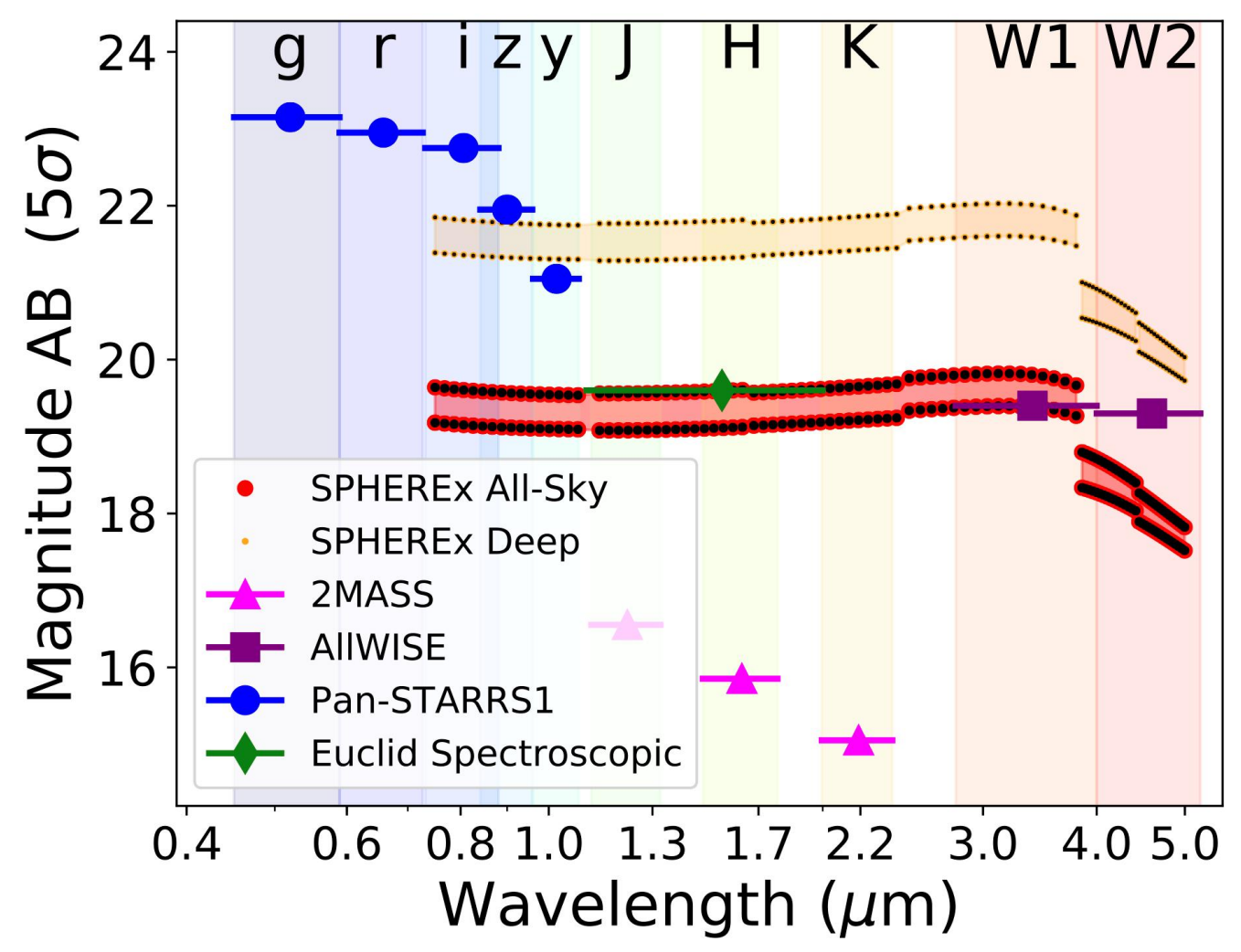

Figure 3. SPHEREx Point source sensitivity in 96 spectral bands compared to other existing and planned surveys.

\section{SPACECRAFT}

The SPHEREx spacecraft, depicted in Figure 4, is designed to elegantly meet the thermal, optical, electrical and operational requirements to produce the all sky survey. The spacecraft bus, which provides power, attitude control, data handling and commanding, is delivered by the Ball Aerospace \& Technologies Corp. from their $\mathrm{BCP}$ series. Detailed design description of the bus is beyond the scope of this paper. Selected engineering considerations for the payload subsystems along with the results of the phase-A risk reduction hardware activities are presented in this section.

\subsection{Telescope}

The SPHEREx optics provide high etendue (A $\Omega$ product), to efficiently map the full sky with high spectroscopic sensitivity, using an off-axis design for stray light control (Figure $5 \mathrm{~A}, \mathrm{~B}$ ). The telescope is a telecentric reflective triplet with a $20 \mathrm{~cm}$ effective aperture, covering a $3.5^{\circ} \times 11.3^{\circ}$ field of view at $\mathrm{f} / 3(60 \mathrm{~cm}$ focal length). Freeform mirrors provide the necessary image quality in a compact low-mass design. A raytrace of the telescope and a cutaway view of the baffle and housing are shown in Figure 5B. Light enters through the baffle, reflects off the primary mirror, goes to the pupil stop at the secondary mirror and reflects off the tertiary mirror to a 


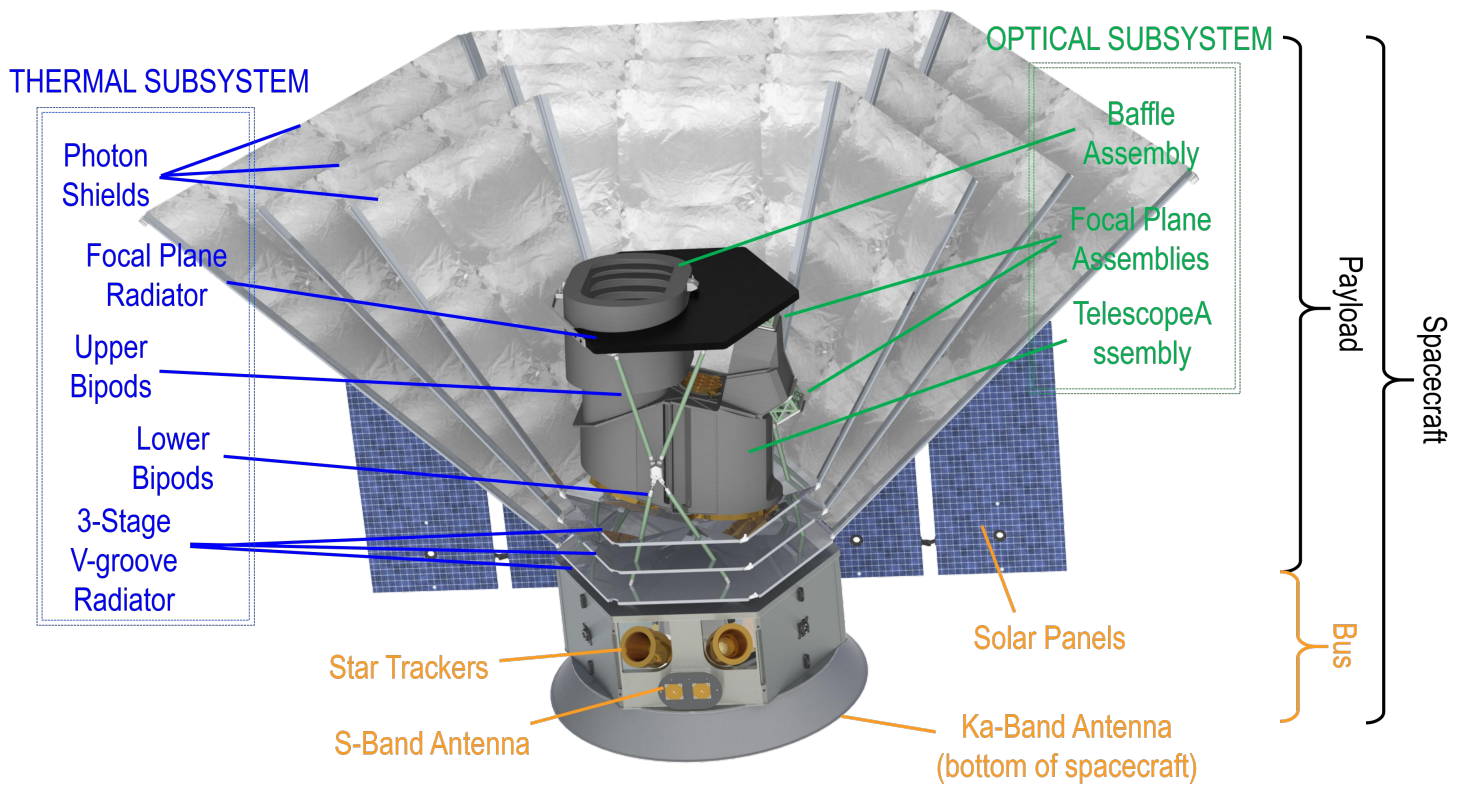

Figure 4. Overview of the SPHEREx spacecraft shown with half the photon shields cut away.

dichroic beam splitter (DBS). The DBS reflects wavelengths shorter than $2.42 \mu \mathrm{m}$ to the three detectors in the $2.5 \mu \mathrm{m}$-cutoff focal plane assembly (FPA) and transmits wavelengths longer than $2.42 \mu \mathrm{m}$ to the three detectors in the $5.3 \mu \mathrm{m}$-cutoff FPA. The back of the DBS (side without the dichroic coating) has a curved surface to compensate for astigmatism and coma generated by focusing through a tilted plate. The optics produces a PSF FWHM $=4.3 "(\mathrm{CBE})$ at $0.75 \mu \mathrm{m}$ including both aberrations and manufacturing tolerance. We manufactured a demonstration mirror of the primary mirror which met the required performance (Figure 5 C,D).

\subsection{Focal Plane Assembly}

The optical subsystem accommodates two FPAs, sharing the FOV through a dichroic beam splitter in reflection and transmission. Each FPA houses three LVF and H2RG detector pairs, with the filter mounted $100 \mu \mathrm{m}$ above each detector. The FPA mosaic plate contains tight-tolerance diamond pins that provide precise optical alignment and stability between the detectors and LVFs, and between the detectors and the focal surface. H2RG coplanarity and LVF to H2RG gaps are tuned by iterating shim thickness with metrology measurements. The LVFs are edge-bonded to a frame with a bondline of $250 \mu \mathrm{m}$. This epoxy and bondline combination are chosen to minimize stress to the joint upon thermal contraction. The FPAs are coupled to the telescope through a re-entrant G10 mount. This provides the thermal isolation required to operate the long wavelength FPA $\sim 20 \mathrm{~K}$ below the telescope housing.

\subsubsection{FPA Thermo-Mechanical Prototype}

We developed a prototype FPA consisting of thermally and mechanically representative LVFs and detectors. The testing demonstrated that the FPA meets SPHEREx mechanical alignment requirements under representative environmental conditions. We assembled the unit shown in Figure 6, carrying out metrology to ensure that the focal surface across the 3 detector arrays could be aligned within the $9 \mu \mathrm{m}$ co-planarity specification. We bonded the LVF substrates into a common frame and verified they were located within $100 \pm 20 \mu \mathrm{m}$ of the detector surface after assembly. We then environmentally tested the FPA by thermally cycling the unit three times from room temperature to $8 \mathrm{~K}$ and exposed the unit to random vibration in 3 axes at qualification levels (10.1 grms for $120 \mathrm{~s}$ ). We carried out metrology between the LVFs and the detector arrays, following each thermal test and before and after vibration testing. The results, summarized in Figure 6, show that the FPA is mechanically stable within SPHEREx specifications. 


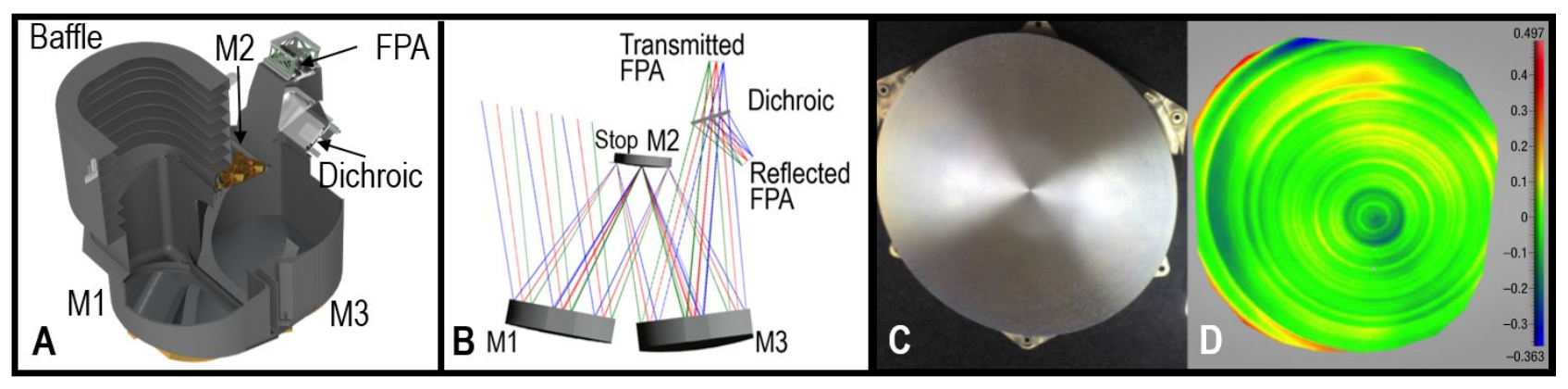

Figure 5. A: Telescope shown with the housing cutaway. Extensive baffling controls stray light. B: Ray trace of the SPHEREx optics. C: Photograph of a free-form mirror demonstration of the SPHEREx flight primary. D: Wavefront error measurement of the prototype with $44 \mathrm{~nm} \mathrm{rms}(0.069$ waves rms at $0.63 \mu \mathrm{m})$, meeting its $80 \mathrm{~nm}$ specification.

\subsubsection{Detectors}

The SPHEREx detectors, located behind the LVFs, are 2048×2048 Hawaii-2RG (H2RG) arrays with $18 \mu$ m pixels produced by Teledyne Sensors Inc. The short-wavelength FPA (Bands 1-3) uses standard $2.5 \mu \mathrm{m}$ cutoff $\mathrm{HgCdTe}$ material; the long-wavelength FPA (Bands 4-6) uses standard $5.3 \mu \mathrm{m}$ cutoff HgCdTe material. SPHEREx's sensitivity (Figure 3) assumes the detectors perform per published specifications (5) with dark current $<0.05$ $e^{-} / s(\mathrm{MEV})$, optical efficiency $>70 \%$, correlated-double-sample (CDS) read noise $<18 e^{-} / s$, and pixel yield $>95 \%$. Delivered arrays typically out-perform these specifications by significant margins (6). The detectors are mature, resulting from significant investment and flight qualification for JWST and Euclid (7), culminating from two decades of advancements for ground-based and space-borne astronomy. The detectors are read out continuously by cold SIDECAR amplifiers manufactured by Teledyne.

\subsubsection{LVFs}

The LVFs provide a spectral band-pass at every position on the focal plane, where the central wavelength of the bandpass increases continuously along one axis. The resolving power $R=\frac{\Delta \lambda}{\lambda}$ is constant over each array; the central wavelength varies as the logarithm of position. This design gives 16 spectral bins per H2RG, and 96 spectral bins in total. The bins are separated by $\Delta \lambda$, in equal steps of 120 pixels or 12.3 arcminutes. The step size is identical for all 6 LVFs. The LVFs also provide the necessary $<0.01 \%$ (OD4) blocking for negligible out-of-band spectral leakage across the wide DBS passband. Viavi Solutions Inc. fabricates the LVFs, based on their experience with similar LVF architectures for New Horizons LEISA and OSIRIS-REx. Viavi has detailed designs for the filters that accommodate padding for spectral overlap between bands and the slight curvature (smile) of the wavelength contours. Table 1 gives the resolution and wavelength values of the bands.

\subsubsection{Band-1 LVF+H2RG prototype}

We developed and tested a flight-compatible LVF procured from Viavi Solutions Inc. that matched the SPHEREx SMEX Band-1 specifications for wavelength range and progression, resolution, and optical efficiency. In a prototype FPA adapted from the CIBER2 design (8), an LVF was mounted above a science-grade H2RG detector. Careful metrology of the mount assembly allowed placement of the filter $100 \mu \mathrm{m}$ above the focal plane, though we installed the LVF with a $525 \mu \mathrm{m}$ gap for the test in order to better characterize LVF-detector reflections. A precise edge on the filter mount aligned to the array using a match-machined assembly jig controlled the lateral registration. We installed the prototype FPA in a LN2 cryostat behind two blocking filters and a Zerodur vacuum window for optical testing.

We measured the spectral response of every pixel in the focal plane by coupling diffuse light from a monochromator to the detector array via an optical fiber and integrating sphere. The measured central wavelengths match the design progression to better than $0.5 \%$. The characteristic "smile" of the iso-wavelength contours, with a curvature of 1.0 to $1.4 \mathrm{~mm}$, closely matches the design used to plan the survey pointings. To quantify ghost reflections between the array and LVF coating layers, we coupled a narrow beam of collimated light to the system 


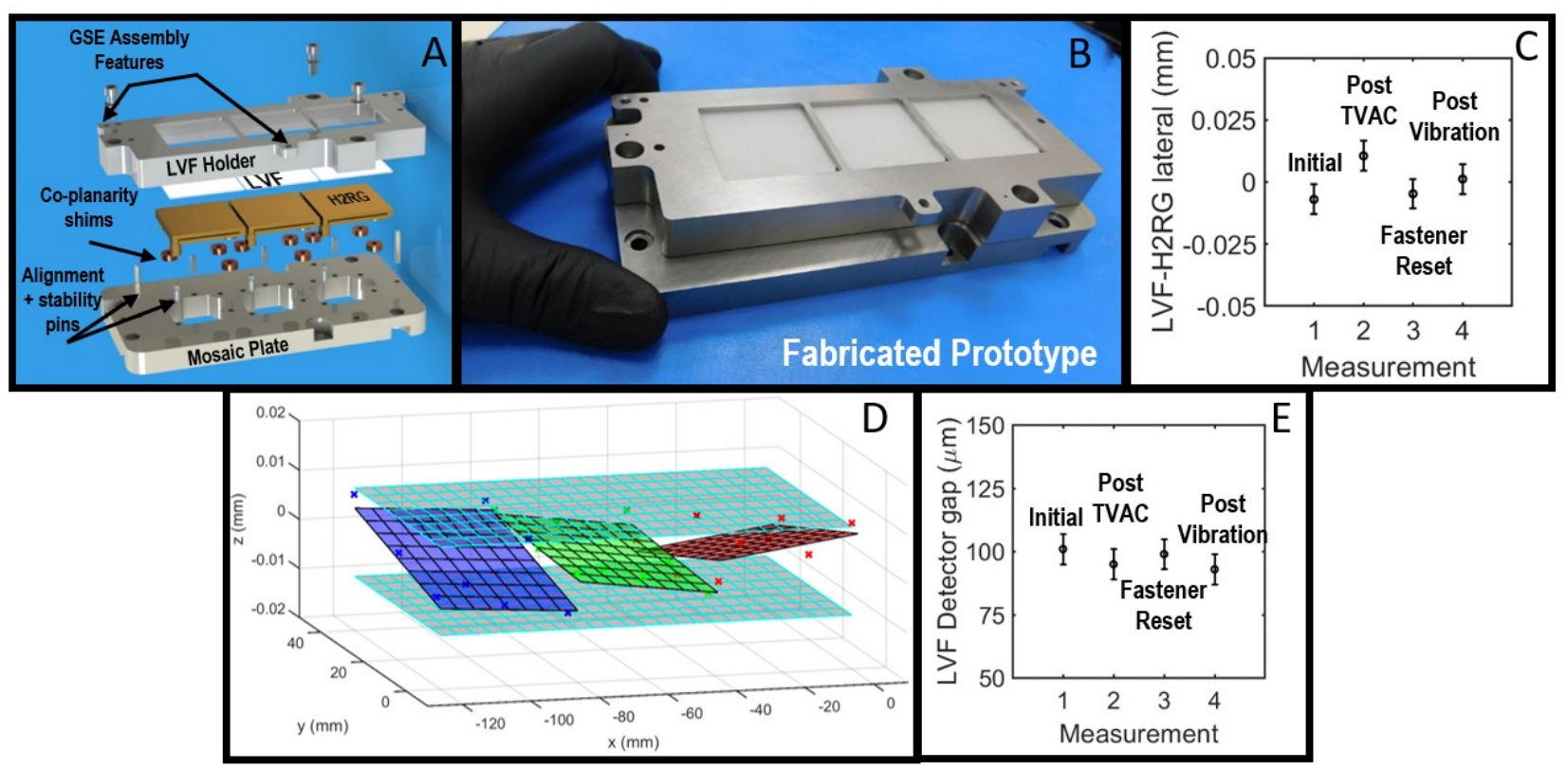

Figure 6. A: Exploded view of the SPHEREx FPA which couples the LVFs to the detectors and maintains all alignments. B: Photograph of the fabricated SPHEREx thermo-mechanical prototype. C: Measured stability of the LVF to detector lateral registration during the environmental testing campaign. We easily meet the $\pm 50 \mu$ m requirement. D: Metrology of the coplanarity of the three H2RG analogs after shimming. The $\pm 9 \mu \mathrm{m}$ requirement indicated by the cyan plane is met. E: Measured stability of the LVF to detector spacing during environmental testing remained stable to within the measurement uncertainty.

Table 1. SPHEREx LVF Band parameters. Note wavelength values are quoted without the spectral padding.

\begin{tabular}{|l|l|l|l|}
\hline Band & $\lambda_{\min }(\mu \mathrm{m})$ & $\lambda_{\max }(\mu \mathrm{m})$ & $\mathrm{R}$ \\
\hline 1 & 0.75 & 1.11 & 41 \\
\hline 2 & 1.11 & 1.64 & 41 \\
\hline 3 & 1.64 & 2.42 & 41 \\
\hline 4 & 2.42 & 3.82 & 35 \\
\hline 5 & 3.82 & 4.42 & 110 \\
\hline 6 & 4.42 & 5.00 & 130 \\
\hline
\end{tabular}

at $9^{\circ}$ angle of incidence. The first ghost reflection carries a relative intensity of $2 \%$, and subsequent ghosts are below $0.1 \%$. Calculated for the $\mathrm{f} / 3$ light cone in the SPHEREx optics with a $100 \mu \mathrm{m}$ gap, these ghost reflections produce a negligible effect, a slight shoulder to the PSF that satisfies the source masking requirement in the EBL deep survey. Note that the measured LVF performance met Viavi's predictions based on the same model used to predict the performance in all of the bands. A photograph of the filter is shown alongside measured wavelength progression and spectral response in Figure 7.

\subsection{Thermal System}

The thermal subsystem radiatively cools the SPHEREx optics and detectors. The passive cooling subsystem has three main elements: a 3-stage V-groove radiator, a deployed series of photon shields, and an FPA radiator for 


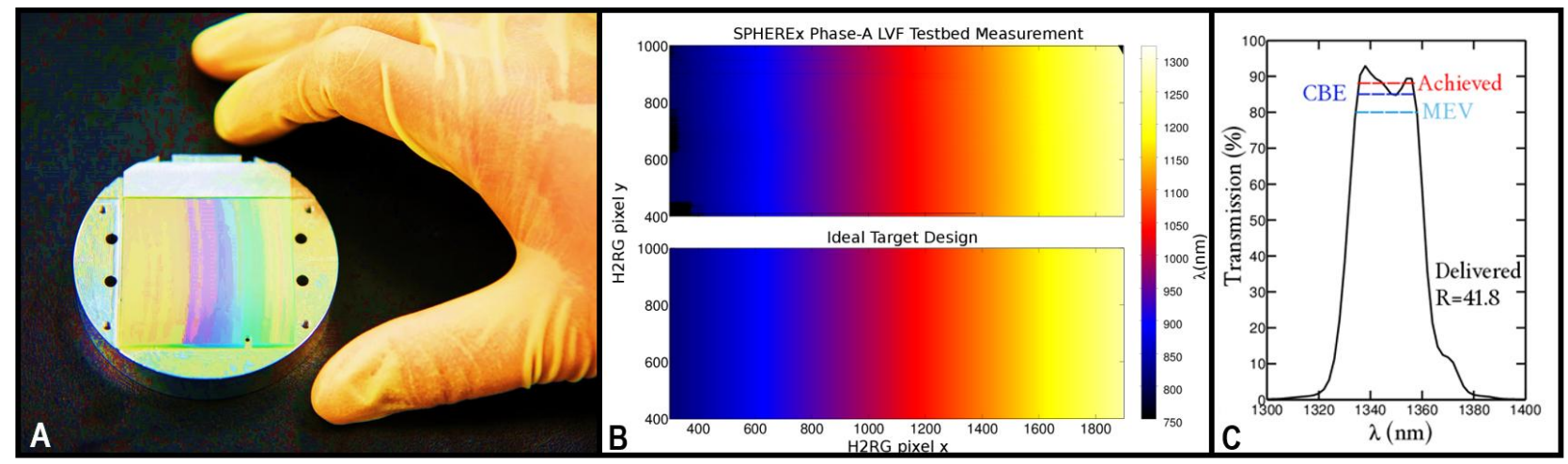

Figure 7. A: Photograph of the fabricated full size SPHEREx Band 1 LVF. B: Measured wavelength progression for the $\mathrm{LVF}+\mathrm{H} 2 \mathrm{RG}$ system tested (top) compared to the ideal design (bottom). Note only the central $11 \times 30 \mathrm{~mm}$ was illuminated in this measurement.C: Measured spectral response function at a single position on the array. Delivered peak transmission and resolution were better than specification.

cooling the $5.3 \mu \mathrm{m}$ detectors. Low-conductivity composite bipods kinematically support and thermally isolate the optical subsystem and FPA radiator. Passive cooling provides robust, long-life, low-mass, and low-cost cooling compared to stored-cryogen or mechanical coolers, but requires proper orbit selection and Earth/Sun avoidance control.

The V-groove cooling system extracts heat conducted through the bipods and cables, and power dissipated by the SIDECARs and detectors. The design is based on the 3-stage V-groove radiator flown on the Planck satellite, where the bipods and low-conductivity cables are thermally strapped in sequence to each V-groove panel, which radiates the conducted heat load to cold space. The conical panels consist of thermally conductive honeycomb, with low-emissivity surfaces to reduce radiative coupling between the stages. Because SPHEREx flies in LEO, we use a nested set of deployed photon shields to protect the optics and V-groove radiators from sunlight, earthshine and thermal re-radiation. The photon shields deploy at progressively larger angles so that radiation from the $\mathrm{V}$-grooves reaches space with a minimum number of reflections.

We analyzed the thermal system with Thermal Desktop V5.8 software, which uses a Monte Carlo based ray-tracing approach, an evolution of the Spitzer and Planck thermal models. The model takes as input heat conducted through the paths through the bipods, cryo-harness, housekeeping wiring, and FPA mount. The dissipated power includes the SIDECARs and FPAs. Figure 8 summarizes the heat flow network. The results from the thermal modeling meet the temperature requirements with large performance margins.

The triple-stage photon shield represents a low-accuracy deployment, common in many high-reliability mechanisms. The shields deploy from a stowed configuration that enable launch vehicle selection flexibility. Each photon shield consists of six gore panels. Each gore attaches to two composite spars through stitched sleeves. The spars in turn mount to the conical V-groove panels at six hinge points. The outer layer incorporates six sprung composite rods between the spar tips to open and control the shape of the deployed layers. Cords connect the three layers, pulling the two inner layers out during deployment. In the stowed state, the middle of the spars mount to the FPA radiator through interlocking snubbers and a restraint cord, providing mechanical support during launch. The shields deploy by activating dual-redundant burn wires that sever the restraint cord by pyrolysis.

\subsubsection{Photon Shield Deployment Prototype}

We fabricated and tested a full-scale mechanical prototype of the photon shields (Figure 9), including hinges and tip spring adapters, and a flight-like burn wire restraint and release mechanism. The subsystem minimized the stowed volume and deployed to the required geometry. During testing, we operated the release system multiple times, measuring the spar tip positions with photogrammetry. The release system demonstrated $100 \%$ 


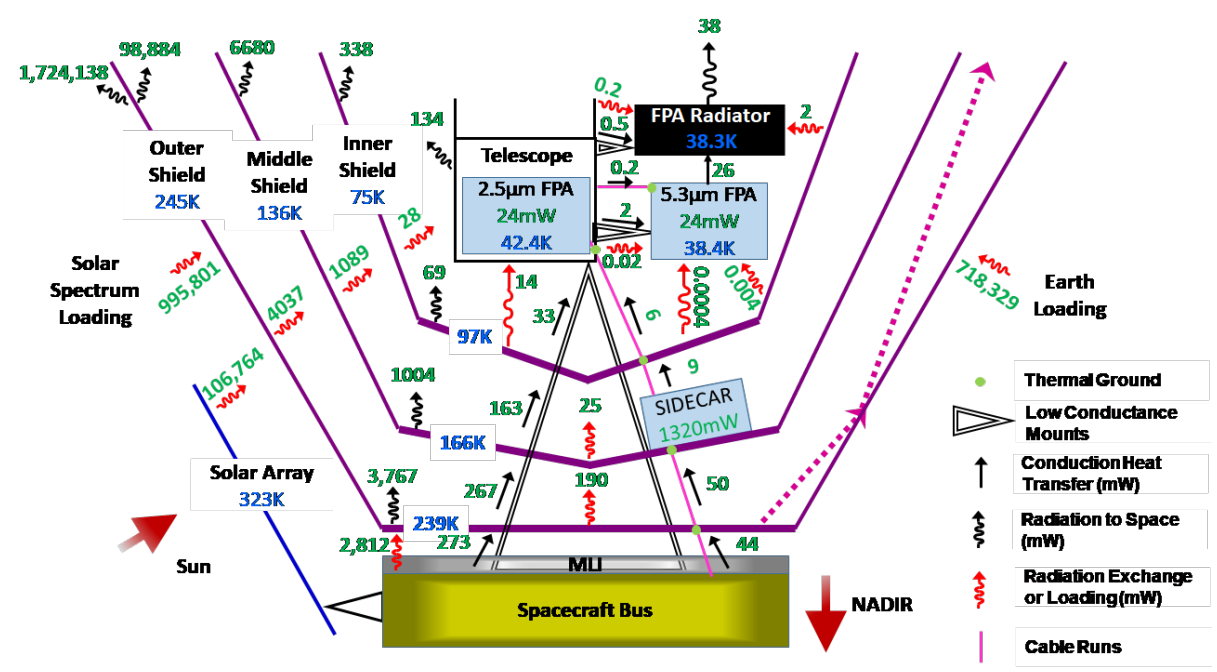

Figure 8. Summary of the radiative, conductive, and dissipative heat loads used in the thermal analysis, with resulting temperatures. The thermal subsystem radiates heat to space via a 3-stage V-groove radiator that extracts conducted and dissipated heat loads. Deployed photon shields protect the cold stages from sunlight and earthshine. The FPA radiator cools the long wavelength FPA. Multi-Layer insulation (MLI) isolates the first stage from the bus

reliability. Figure 9 gives the position of the tips over multiple tests, both before and after vibration testing, meeting the $<40 \mathrm{~mm}$ radius positional requirement. We measured the ripple in one of the deployed gores with photogrammetry to be better than $\pm 2.4^{\circ} \mathrm{RMS}$, well within the required $\pm 15^{\circ}$ peak ripple.

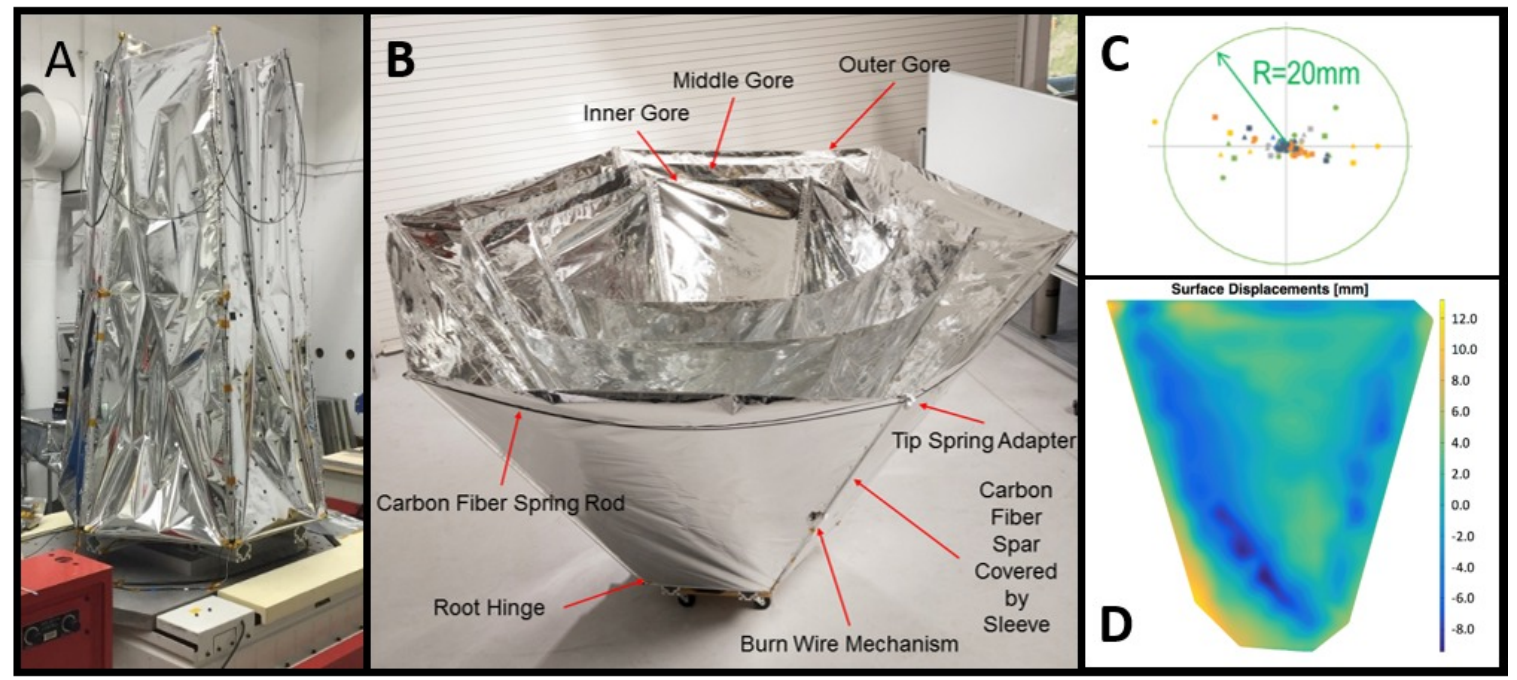

Figure 9. Photon shield deployment demonstration hardware in the JPL Advanced Large Precision Structures (ALPS) Laboratory, and measured flight-like performance results. A: The stowed photon shield system during vibration testing. B: The deployed photon shield met the deployment requirements with significant margin after vibration testing. C: The shield tips deploy to within $4.8 \mathrm{~mm} \mathrm{rms}$ of the required position, well within the $<40$ mm radius requirement. We compiled these measurements over 20 deployments for the 18 tip locations before and after vibration testing. D: Measured surface of an outer gore, giving $2.4^{\circ} \mathrm{rms}$ large-scale ripple, smoother than the required $\pm 15^{\circ}$ peak ripple

We vibration tested the shields in stowed configuration with representative mechanical interfaces. When stowed, the release mechanism retains the spars against the FPA radiator, and the blankets are not under 
tension but loosely folded for launch. The deployed shape after vibration testing was within $0.5 \mathrm{~mm}$ of the pre-vibe surface. Deployment testing after vibration demonstrated that the restraint/release system is robust to launch loads. The mechanism showed no hysteretic or yield effects, and the burn wire loop was in positive contact with the release cord after test. We repeated metrology on the gore surface after vibration and confirmed the surface remained specular and stable, within the required ripple.

\subsubsection{Sub-scale Thermal Prototype}

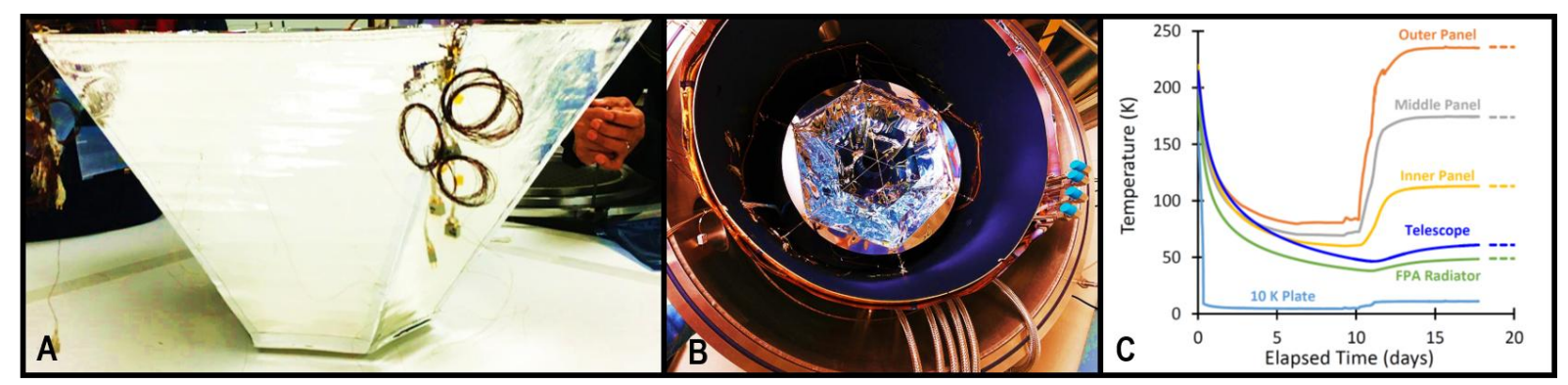

Figure 10. A: Photograph of the subscale thermal prototype viewing the outer photon shield with instrumentation wiring visible. B: Subscale thermal prototype installed in the cryogenic chamber at JPL. C: Temperatures during the environmental testing at JPL. Solar and terrestrial thermal load simulators were activated on day 10 and temperatures were stabilized by day 16 to within $1 \mathrm{~K}$ of model predictions.

Testing a sub-scale thermal model captures the essential features of a passive system without the time and expense of a full-scale test (9). We developed a sub-scale prototype for cryogenic testing (10) that accurately replicates the geometry of the full-scale V-groove radiator and photon shield assembly (Figure 10). The prototype is approximately $\frac{1}{4}$ scale, and non-scaled differences to flight system are second order and small compared with the heat load margins in the thermal design. The modeled performance of the scaled prototype closely matches measurements, which validates the thermal modelling for the flight system.

We tested the prototype in a thermal vacuum chamber in the JPL Advanced Thermal Technology Lab. A shroud cooled to $77 \mathrm{~K}$ surrounded the prototype, with an infrared-absorbing plate cooled to $10 \mathrm{~K}$ placed in front of the shields to simulate cold space. The prototype rapidly cooled down to operating temperatures. We matched the flight radiation loads by operating resistive heaters embedded in the outer photon shield. We also applied heater power to simulate the dissipated loads from the detectors on the telescope and FPA radiator, and the SIDECARs on the middle conical panel. The temperatures equilibrated to values near the prototype model predictions within $1 \mathrm{~K}$.

\section{CONCLUSIONS}

The SPHEREx mission will provide a rich spectral dataset for the general astronomical community to mine for the coming decades. It provides high-impact results in fields ranging from Solar system studies to high redshift quasars, in addition to three primary goals. If selected by NASA in the upcoming MIDEX decision, SPHEREx will be slated for launch in 2023. This timing provides an excellent opportunity for profound synergies, identifying targets for deep spectroscopic follow-up with flagship missions such as JWST. Having undergone extensive design studies and prototyping during Phase-A, the mission architecture and design is mature and ready for implementation.

\section{ACKNOWLEDGMENTS}

We'd like to thank the contributors at L3-SSG, VIAVI, Sierra-Lobo and Photon Engineering, who helped develop this robust design. Chris Smith and Myrtle Lin were essential in the development of the FPA prototype. Part of the research described in this paper was carried out at the Jet Propulsion Laboratory, California Institute of Technology, under a contract with the National Aeronautics and Space Administration. 


\section{REFERENCES}

[1] Doré, O., Werner, M. W., Ashby, M., Banerjee, P., Battaglia, N., Bauer, J., Benjamin, R. A., Bleem, L. E., Bock, J., Boogert, A., Bull, P., Capak, P., Chang, T.-C., Chiar, J., Cohen, S. H., Cooray, A., Crill, B., Cushing, M., de Putter, R., Driver, S. P., Eifler, T., Feng, C., Ferraro, S., Finkbeiner, D., Gaudi, B. S., Greene, T., Hillenbrand, L., Höflich, P. A., Hsiao, E., Huffenberger, K., Jansen, R. A., Jeong, W.-S., Joshi, B., Kim, D., Kim, M., Kirkpatrick, J. D., Korngut, P., Krause, E., Kriek, M., Leistedt, B., Li, A., Lisse, C. M., Mauskopf, P., Mechtley, M., Melnick, G., Mohr, J., Murphy, J., Neben, A., Neufeld, D., Nguyen, H., Pierpaoli, E., Pyo, J., Rhodes, J., Sandstrom, K., Schaan, E., Schlaufman, K. C., Silverman, J., Su, K., Stassun, K., Stevens, D., Strauss, M. A., Tielens, X., Tsai, C.-W., Tolls, V., Unwin, S., Viero, M., Windhorst, R. A., and Zemcov, M., "Science Impacts of the SPHEREx All-Sky Optical to NearInfrared Spectral Survey: Report of a Community Workshop Examining Extragalactic, Galactic, Stellar and Planetary Science," ArXiv e-prints 1606.07039 (June 2016).

[2] Doré, O., Werner, M. W., Ashby, M. L. N., Bleem, L. E., Bock, J., Burt, J., Capak, P., Chang, T.-C., ChavesMontero, J., Charbonneau, D., Chen, C. H., Civano, F., Cleeves, I. I., Cooray, A., Crill, B., Crossfield, I. J. M., Cushing, M., de la Torre, S., DiMatteo, T., Dvory, N., Dvorkin, C., Espaillat, C., Eisenstein, D., Ferraro, S., Finkbeiner, D., Greene, J., Hewitt, J., Hogg, D. W., Huffenberger, K., Jun, H.-S., Ilbert, O., Jeong, W.-S., Johnson, J., Kim, M., Kirkpatrick, J. D., Kowalski, T., Korngut, P., Li, J., Lisse, C. M., MacGregor, M., Mamajek, E. E., Mauskopf, P., Melnick, G., Ménard, B., Neyrinck, M., Öberg, K., Pisani, A., Rocca, J., Salvato, M., Schaan, E., Scoville, N. Z., Song, Y.-S., Stevens, D. J., Tenneti, A., Teplitz, H., Tolls, V., Unwin, S., Urry, M., Wandelt, B., Williams, B. F., Wilner, D., Windhorst, R. A., Wolk, S., Yorke, H. W., and Zemcov, M., "Science Impacts of the SPHEREx All-Sky Optical to Near-Infrared Spectral Survey II: Report of a Community Workshop on the Scientific Synergies Between the SPHEREx Survey and Other Astronomy Observatories," ArXiv e-prints, 1805.05489 , arXiv:1805.05489 (May 2018).

[3] Spangelo, S. C., Katti, R. M., Unwin, S. C., and Bock, J. J., "All-sky survey mission observing scenario strategy," Journal of Astronomical Telescopes, Instruments, and Systems 1, 037001 (June 2015).

[4] Schlegel, D. J., Finkbeiner, D. P., and Davis, M., "Maps of Dust Infrared Emission for Use in Estimation of Reddening and Cosmic Microwave Background Radiation Foregrounds," The Astrophysical Journal 500, 525-553 (June 1998).

[5] Teledyne, "H2rg visible and infrared focal plane array. teledyne imaging sensors, http://www.teledynesi.com/products/documents/h2rg(2017).

[6] Blank, R., Anglin, S., Beletic, J. W., Bhargava, S., Bradley, R., Cabelli, C. A., Chen, J., Cooper, D., Demers, R., Eads, M., Farris, M., Lavelle, W., Luppino, G., Moore, E., Piquette, E., Ricardo, R., Xu, M., and Zandian, M., "H2RG focal plane array and camera performance update," in [High Energy, Optical, and Infrared Detectors for Astronomy V. Proceedings of the SPIE, Volume 8453, article id. 845310, $16 \mathrm{pp}$. (2012).], 8453 (July 2012).

[7] Secroun, A., Serra, B., Clémens, J. C., Legras, R., Lagier, P., Niclas, M., Caillat, L., Gillard, W., Tilquin, A., Ealet, A., Barbier, R., Ferriol, S., Kubik, B., Smadja, G., Prieto, E., Maciaszek, T., and Norup Sorensen, A., "Characterization of H2RG IR detectors for the Euclid NISP instrument," in [Proceedings of the SPIE, Volume 9915, id. 99151 Y 9 pp. (2016).], 9915, 99151Y (July 2016).

[8] Lanz, A., Arai, T., Battle, J., Bock, J., Cooray, A., Hristov, V., Korngut, P., Lee, D. H., Mason, P., Matsumoto, T., Matsuura, S., Morford, T., Onishi, Y., Shirahata, M., Tsumura, K., Wada, T., and Zemcov, M., "Studying extragalactic background fluctuations with the Cosmic Infrared Background ExpeRiment 2 (CIBER-2)," in [Proceedings of the SPIE, Volume 9143, id. $91433 N 10$ pp. (2014).], 9143, 91433N (Aug. 2014).

[9] DiPirro, M., Tuttle, J., Ollendorf, S., Mattern, A., Leisawitz, D., Jackson, M., Francis, J., Hait, T., Cleveland, P., Muheim, D., and Mastropietro, A. J., "High-fidelity cryothermal test of a subscale large space telescope," in [Cryogenic Optical Systems and Instruments XII. Edited by Heaney, James B.; Burriesci, Lawrence G.. Proceedings of the SPIE, Volume 6692, article id. 669202 (2007).], 6692 (Sept. 2007).

[10] Bolton, D., ed., [Spectro-Photometer for the History of the Universe, Epoch of Reionization, and Ices Explorer, One Quarter Scale Prototype Thermal Testing], 0 0, The 47th interational Conference on Environmental Systems, The 47th interational Conference on Environmental Systems, The address of the publisher (7 2017). An optional note. 